Avendaño Arrazate, C.H., Campos Rojas, E., López Palestina, C.U., Martínez Bolaños, M., Caballero Pérez, J.F., Báez Alonso, M., Ariza Flores, R., \& Cadena Iñiguez, J. (2021). Actividad antioxidante en genotipos de Theobroma spp. (Malvaceae) en México. Revista de Biología Tropical, 69(2), 507-523. DOI 10.15517/rbt.v69i2.41626

DOI 10.15517/rbt.v69i2.41626

\title{
Actividad antioxidante en genotipos de Theobroma spp. (Malvaceae) en México
}

\author{
Carlos Hugo Avendaño Arrazate ${ }^{1}$ \\ Eduardo Campos Rojas ${ }^{2}$ \\ Cesar Uriel López Palestina ${ }^{3}$ \\ Misael Martínez Bolaños ${ }^{1}$ \\ Juan Francisco Caballero Pérez ${ }^{1}$ \\ Moisés Báez Alonso ${ }^{1}$ \\ Rafael Ariza Flores ${ }^{4}$ \\ Jorge Cadena Iñiguez ${ }^{5 *}$
}

1. Instituto Nacional de Investigaciones Forestales, Agrícolas y Pecuarias (INIFAP)-Campo Experimental Rosario Izapa, km 18, Carretera Tapachula-Cacahoatán, Tuxtla chico, C.P. 30870, Chiapas, México; avendano.carlos@inifap.gob.mx,martinez.misael@inifap.gob.mx, caballero.juan@inifap.gob.mx, baez.moises@inifap.gob.mx

2. Universidad Autónoma Chapingo-Departamento de Fitotecnia, C.P. 56230, Chapingo, Estado de México; educamro55@gmail.com

3. Instituto de Ciencias Agropecuarias, Universidad Autónoma del estado de Hidalgo, Rancho Universitario, Av. Universidad km 1, Ex-Hacienda de Aquetzalpa AP 32, C.P. 43600, Tulancingo, Hidalgo, México; cesar_lopez@uaeh.edu.mx

4. INIFAP-Campo Experimental de Iguala, km 2.5 Carretera Igual-Tuxpan, C.P. 4100, Iguala, Guerrero, México; ariza.rafael@inifap.gob.mx

5. Postgrado de Innovación en Manejo de Recursos Naturales Colegio de Posgraduados, Campus San Luis Potosí, Iturbide 73, Salinas de Hidalgo, C.P. 78620, San Luis Potosí, México; jocadena@gmail.com (*Correspondencia).

Recibido 30-IV-2020. Corregido 17-II-2021. Aceptado 05-III-2021.

\section{ABSTRACT \\ Antioxidant activity in genotypes of Theobroma spp. (Malvaceae) in Mexico}

Introduction: The organoleptic qualities of aromatic species and their derived products are directly related to some characteristics of flavor, color and nutritional value and depend largely on their genetic origin and content of secondary metabolites. Objective: the antioxidant activity of different genotypes of Theobroma spp. from Mexico was evaluated in order to distinguish promising qualities for genetic improvement, and to differentiate phylogenetic traits, considering biochemical variables. Methods: the amount of phenols, flavonoids and antioxidant activity was determined by ABTS and DDPH, in addition to the content of anthocyanins, theobromine and caffeine in four species of Theobroma L., and 50 genotypes derived from $T$. cacao. The results were analyzed using an analysis of variance, means test, principal component analysis and cladistic analysis. Results: there are highly significant differences between genotypes. The phenol content ranged from $7.5-85 \mathrm{mg} \mathrm{g}^{-1}$; flavonoids 6.57-69.6 $\mathrm{mg} \mathrm{g}^{-1}$, antioxidant activity by ABTS of 17.3-86.1 and by DDPH of 40.0-53.3; anthocyanin content of 0.01-3, caffeine of 1.8-6.7-and theobromine of 2.9-9.8 $\mathrm{mg} \mathrm{g}^{-1}$. Principal component and cladistic analysis helped explain the variation found and distinguish evolutionary characters and phylogenetic brotherhoods. The variation in content of phenols, flavonoids, antioxidant activity, anthocyanins, theobromine and caffeine was mainly 


\begin{abstract}
due to the degree of domestication, while for the group of genotypes derived from $T$. cacao (forastero, trinitario and criollo) it was the origin of the seeds. Conclusions: the degree of domestication influences the content of phenols and antioxidant capacity. The results suggest that the evaluated variables can help to form criteria for genetic improvement in the complex derived from T. cacao oriented to the selection of higher phenol content and greater antioxidant activity.
\end{abstract}

Key words: phenols; theobromine; forastero; trinitario; criollo.

A nivel mundial se reconocen tres tipos $\mathrm{u}$ orígenes genéticos de cacao (Theobroma cacao L.): el Criollo que presenta cotiledón de color blanco y es poco cultivado por ser susceptible a enfermedades; el Forastero, de la región del Amazonas; y el Trinitario, un hibrido entre el tipo Forastero y el Criollo (Vázquez-Ovando, Molina-Freaner, Nuñez-Farfán, BetancurAncona, \& Salvador-Figueroa, 2015). El tipo Forastero presenta cotiledón de color púrpura debido a las antocianinas, es en general el más cultivado y registra mayor resistencia a enfermedades (Afoakwa, 2010). En México se cultivan 59555 ha de T. cacao, con una producción de 28473 toneladas y un rendimiento promedio de $486 \mathrm{~kg} \mathrm{ha}^{-1}$ (Servicio de Información Agroalimentaria y Pesquera, 2020); con un consumo per cápita de $0.5 \mathrm{~kg}$. La producción nacional de cacao satisface únicamente el $41.2 \%$ de la demanda interna y su cultivo es una alternativa de desarrollo rural, sin dañar el ambiente debido a que se cultiva bajo sistemas agroforestales (Suárez-Venero, Avendaño-Arrazate, Ruíz-Cruz, \& Estrada-de los Santos, 2019). En cuanto a variedades cultivadas, en México se cuenta con los tres tipos genéticos de T. cacao (forastero, trinitario y criollo).

Los cacaos de México se caracterizan por su calidad y aroma (tipo criollo) y es demandado por la industria que elabora chocolate de alta gama (Avendaño-Arrazate, Martínez-Bolaños, \& Mendoza-López, 2018). Sin embargo, es importante realizar estudios con mayor número de variantes biológicas de Theobroma spp., que facilite diferenciarlos por su carácter nutracéutico, por ejemplo, su actividad antioxidante, lo cual podría aumentar su valor en el mercado internacional (Ramírez, Cely, \& Ramírez, 2013).
La actividad antioxidante inducida por los ácidos fenólicos y flavonoides en los genotipos de cacao (Theobroma spp.), se relaciona directamente con algunas características del sabor, color, palatabilidad y valor nutricional (Padilla, Rincón, \& Bou-Rached, 2008), los cuales tienen que ver con posibles efectos beneficiosos para la salud. Lo anterior se debe a que los antioxidantes desactivan radicales libres, minimizando el daño y protegiendo al organismo de enfermedades cardiovasculares, degenerativas, y desarrollo de cáncer (Abbe \& Amin, 2008). Autores como Hii, Law, Suzannah, y Cloke (2009), mencionan que los polifenoles, han ganado interés debido a su actividad antioxidante y se asocian a efectos anticancerígenos, aterogénicos, antiulcerosos, antitrombóticos, antiinflamatorios, inmunomoduladores, antimicrobianos, vasodilatadores y analgésicos. Otros autores como Valadez-Carmona et al. (2017) mencionan que la cáscara de cacao contiene polifenoles y registra actividad antioxidante de hasta $323.7 \pm 26.5 \mathrm{mg}$ de ácido gálico equivalente (GAE)/100 g de biomasa seca.

Pallares-Pallares, Perea-Villamil, y LópezGiraldo (2016), reportaron en la variedad de cacao CCN-51 (tipo forastero) que, de las etapas de beneficio del cacao, la fermentación es la que más impacta en el contenido de polifenoles totales y su actividad antioxidante, ya que durante el manejo postcosecha, los granos son sometidos a diferentes etapas que pueden afectar el contenido de polifenoles; sin embargo, esto es crucial para el desarrollo de la calidad organoléptica (Zapata-Bustamante, TamayoTenorio, \& Alberto-Rojano, 2013).

De acuerdo con Abbe y Amin (2008) los factores que afectan la cantidad y calidad de polifenoles en las semillas de cacao y subproductos (chocolate) son las condiciones 
agroclimáticas del sitio de cultivo, la variedad, condiciones de fermentación, proceso de manufactura y biodisponibilidad de los polifenoles. Niemenak, Rohsiusb, Elwersb, Ndoumoua, y Liebereib (2006), señalan que la fermentación y el secado del cacao son pasos críticos en su procesamiento, ya que causan degradación de antocianinas, lo cual modifica el color de los cotiledones en los granos, transformándolos mediante oxidación hacia quinonas por las polifenol-oxidasas, reduciendo la astringencia y desarrollo del color marrón (Crozier et al., 2011).

Investigaciones con humanos han mostrado el efecto de los polifenoles del cacao en los niveles lipídicos, como una disminución en el colesterol total y regulación en la presión arterial (Zapata-Bustamante et al., 2013). Otros autores como Cádiz-Gurrea et al. (2014) aislaron e identificaron en extractos de $T$. cacao 61 compuestos relacionados con polifenoles, y evaluaron su actividad antioxidante observando reducción de especies reactivas de oxígeno.

El cacao y sus productos derivados (cacao en polvo, licor de cacao, manteca y chocolates) contienen polifenoles con diferentes niveles potenciales antioxidantes (Fisher, Hughes, Gerhard-Herman, \& Hollenberg, 2003; Othman, Mail, Abdul, \& Adenan, 2007), incluso, por su riqueza, registran mayor actividad antioxidante en comparación con los tés (Camelia sinensis) y el vino tinto. En México se distribuyen de manera natural las especies de T. cacao y $T$. bicolor (Gálvez-Marroquín et al., 2016); pero, no existen estudios que ayuden a diferenciar en estas y otras especies de Theobroma spp. dichas características bioquímicas y funcionales, principalmente en $T$. bicolor que está teniendo relevancia en la elaboración de chocolates y bebidas tradicionales en combinación con cacaos criollos de T. cacao (McCoy et al., 2019). Por lo anterior, se evaluó la actividad antioxidante de diferentes genotipos de cacao (Theobroma spp.) con el fin de distinguir el de mayor contenido de metabolitos secundarios relacionados con actividad antioxidante, diferenciando rasgos filogenéticos que coadyuven en corto plazo al mejoramiento genético de nuevos cultivares.

\section{MATERIALES Y MÉTODOS}

La actividad antioxidante se evaluó en genotipos de las especies silvestres T. mammosum y T. angustifolium, además de la especie semi domesticada $T$. bicolor y la domesticada T. cacao, contribuyendo esta última con 50 variantes (forastero, trinitario y criollo), haciendo un total de $\mathrm{N}=53$. Todos los genotipos fueron árboles maduros en producción con rango de edad de 10-20 años procedentes del Campo Experimental Rosario Izapa-INIFAP de México (Tabla 1). El estudio se realizó en $\mathrm{N}=$ 250 semillas que fueron extraídas del fruto en madurez fisiológica, lavadas y secadas a pleno sol hasta que alcanzaron una humedad de $7 \%$.

Extracción de polifenoles totales: El contenido total de fenoles se determinó mediante el método modificado por Waterman y Mole (1994). A partir de la trituración de semillas secas de cacao. Mediante el fraccionamiento de un extracto de cacao obtenido en una solución acuosa de metanol al $70 \%$ y precipitaciones selectivas con gelatina, se separaron compuestos fenólicos. Se colocaron $0.75 \mathrm{~mL}$ de solución acuosa de metanol al $70 \%$ y se mezcló en un agitador magnético a temperatura ambiente durante $45 \mathrm{~min}$, para posteriormente filtrar el extracto a través de papel Whatman No. 4 y aforar a $100 \mathrm{~mL}$ en un matraz volumétrico, con solución acuosa de metanol al $70 \%$ (extracto crudo). La concentración de estas fracciones se determina luego de purificar sobre polivinilpirrolidina insoluble (PVP) usando el método colorimétrico del reactivo de Folin Ciocalteu y un espectrofotómetro UV-VIS a una longitud de onda de $760 \mathrm{~nm}$ (Singleton \& Rossi, 1965). Para la determinación de los polifenoles totales se trasfirió $5 \mathrm{~mL}$ de extracto crudo a un matraz volumétrico de $50 \mathrm{~mL}$ aforado con agua ultrapura. El cálculo de fenoles totales realizó mediante la ecuación:

PT = A equivalentes de ácido gálico - B equivalentes de ácido gálico 
TABLA 1

Fenotipos de cuatros especies de Theobroma evaluados en su actividad antioxidante

TABLE 1

Phenotypes of four Theobroma species evaluated for their antioxidant activity

\begin{tabular}{|c|c|c|c|c|c|}
\hline Código & Genotipo & Origen genético & Código & Genotipo & Origen genético \\
\hline $\mathrm{F} 1$ & CATIE R1 & Forastero & $\mathrm{T} 8$ & RIM 88 & Trinitario \\
\hline $\mathrm{F} 2$ & CATIE R4 & Forastero & T9 & RIM 105 & Trinitario \\
\hline F3 & CATIE R6 & Forastero & $\mathrm{T} 10$ & RIM 117 & Trinitario \\
\hline $\mathrm{F} 4$ & CC-137 & Forastero & C9 & CNF-1 & Criollo \\
\hline F5 & ICS 95 & Forastero & $\mathrm{C} 10$ & $\mathrm{CNF}-2$ & Criollo \\
\hline F6 & PMCT 58 & Forastero & C11 & $\mathrm{CNF}-3$ & Criollo \\
\hline $\mathrm{T} 1$ & Regalo de Dios & Trinitario & $\mathrm{C} 12$ & CNF-4 & Criollo \\
\hline $\mathrm{C} 1$ & Lacandón & Criollo & $\mathrm{C} 13$ & $\mathrm{CNF}-5$ & Criollo \\
\hline F7 & CAERI-1 & Forastero & $\mathrm{C} 14$ & CNF-6 & Criollo \\
\hline F8 & CAERI-2 & Forastero & $\mathrm{C} 15$ & P-49 & Criollo \\
\hline $\mathrm{C} 2$ & Verde Gustavo & Criollo & $\mathrm{C} 16$ & P-208 & Criollo \\
\hline $\mathrm{C} 3$ & Rojo Gustavo & Criollo & $\mathrm{C} 17$ & Catongo & Criollo \\
\hline $\mathrm{C} 4$ & Rojo Samuel & Criollo & $\mathrm{C} 18$ & Blanco Marfil & Criollo \\
\hline $\mathrm{C} 5$ & Lagarto & Criollo & F13 & UF-273 & Forastero \\
\hline C6 & Carmelo & Criollo & F14 & PA-169 & Forastero \\
\hline $\mathrm{C} 7$ & Cuyul & Criollo & C19 & Lagartito & Criollo \\
\hline F9 & CAERI-4 & Forastero & $\mathrm{C} 20$ & Tuzantan 2 & Criollo \\
\hline F10 & CAERI-5 & Forastero & T11 & Tuzantan 3 & Trinitario \\
\hline F11 & SCA-6 & Forastero & Tma & T. mammosum & T. mammosum \\
\hline F12 & SCA-12 & Forastero & Tan & T. angustifolium & T. angustifolium \\
\hline $\mathrm{C} 8$ & Porcelana & Criollo & $\mathrm{C} 21$ & LAC-PT-01 & Criollo \\
\hline $\mathrm{T} 2$ & CAER-6 & Trinitario & $\mathrm{C} 22$ & LAC-PT-08 & Criollo \\
\hline $\mathrm{T} 3$ & CAERI-7 & Trinitario & Tbi & Pataxte & T. bicolor \\
\hline $\mathrm{T} 4$ & CAERI-8 & Trinitario & $\mathrm{T} 12$ & RO-62-018B & Trinitario \\
\hline T5 & RIM 24 & Trinitario & $\mathrm{T} 13$ & RO-62 018A & Trinitario \\
\hline T6 & RIM 44 & Trinitario & $\mathrm{T} 14$ & Arcoíris & Trinitario \\
\hline $\mathrm{T} 7$ & RIM 56 & Trinitario & & & \\
\hline
\end{tabular}

Capacidad de inhibir radical libre 1,1-difenil-2-picrilhidrazil (DPPH): Con base en la metodología propuesta por BrandWilliams, Cuvelier, y Berset (1995), se prepararon $10 \mathrm{~mL}$ de solución stock de DPPH a $1 \mathrm{mM}$ en metanol (99\%) de pureza, se agito hasta la solubilización completa del compuesto y se almaceno a $4{ }^{\circ} \mathrm{C}$ protegido de la luz. A partir de esta solución stock se prepararon 50 $\mathrm{mL}$ de DPPH a $20 \mu \mathrm{M}$ en metanol (99\%) de pureza. Para la inhibición del radical DPPH en las distintas muestras se realizó el filtrado y centrifugado a $10000 \mathrm{rpm} / 10 \mathrm{~min}$ a $4{ }^{\circ} \mathrm{C}$ de 1.0 $\mathrm{g}$ de semilla molida de caco. Posteriormente en una cubeta de cuarzo se adicionaron $25 \mu \mathrm{L}$ de la solución trabajo y $975 \mu \mathrm{L}$ de solución DPPH a $100 \mu \mathrm{M}$. Se realizaron lecturas en espectrofotómetro de Uv/VIS a una longitud de onda de $517 \mathrm{~nm}$, previo a un reposo de la muestra por $30 \mathrm{~min}$. Los resultados se expresan en $\mathrm{mg}$ equivalentes de ácido ascórbico (EAA) $100 \mathrm{~g}^{-1}$.

Capacidad de inhibir radical libre 2,2-azinobis (3-etilbenzotiazolino-6-ácidosulfonico) $\left(\mathrm{ABTS}^{\circ}+\right.$ ): El radical $\mathrm{ABTS}^{\circ}+$ se forma tras la reacción de ABTS $(7 \mathrm{mM})$ ABTS (Sigma-Aldrich) con persulfato potásico $\left(\mathrm{K}_{2} \mathrm{~S}_{2} \mathrm{O}_{8}\right)(140 \mathrm{mM}$, concentración final) 
incubados a temperatura ambiente y en oscuridad durante $16 \mathrm{~h}$. Una vez formado el radical $\mathrm{ABTS}^{\circ}+$ se diluyó con metanol hasta obtener un valor de absorbancia entre 0.7 a 1.2. Para la inhibición del radical ABTS en las distintas muestras se realizó el filtrado y centrifugado 10 $000 \mathrm{rpm} / 10 \mathrm{~min}$ a $4{ }^{\circ} \mathrm{C}$. Luego en una cubeta de poliestireno se adicionaron $10 \mu \mathrm{L}$ de la solución de trabajo y $990 \mu \mathrm{L}$ del radical ABTS. La disminución de la absorbancia se registró a 734 $\mathrm{nm}$ por espacio de $10 \mathrm{~min}$. Los resultados se expresan en mg equivalentes de ácido ascórbico (EAA) $100 \mathrm{~g}^{-1}$ peso fresco (Re et al., 1999).

Pigmentación antociánicos: Se adapto una metodología de análisis para determinar el contenido total de antocianinas en cacao utilizando espectrofotometría Uv-Vis y tomando como referencia el método colorimétrico, para determinar los pigmentos del cacao descrito en la International Office of Cocoa, Chocolate and Sugar Confectionery (Hasing, 2004). Los pigmentos antocianicos del cacao fueron extraídos con una solución de Alcohol n-amílico acidificado con HCL $0.1 \mathrm{~N}$ y posteriormente cuantificados por espectrofotometría Uv-VIS a $544 \mathrm{~nm}$. Las semillas secas, se pesaron con precisión de $0.1 \mathrm{mg}$, y $1.0 \mathrm{~g}$ del material seco y no desengrasado se molió en un mortero. Se añadieron $2 \mathrm{~mL}$ de ácido clorhídrico $0.1 \mathrm{~N}$ utilizando en una bureta que contenía $20 \mathrm{~mL}$ de ácido. Se añadió el ácido restante y dejó reposar por una hora, mezclando ocasionalmente. Posteriormente se transfirió el contenido del mortero a un tubo de centrifuga, sin enjugar el mortero, se centrifugo por $10 \mathrm{~min}$ a una velocidad de 3 $000 \mathrm{rpm}$. Se tomaron $10 \mathrm{~mL}$ del sobrenadante del extracto ácido con una pipeta volumétrica y se transfirió a un tubo de ensayo graduado y se adicionaron $10 \mathrm{~mL}$ de alcohol n-amilico saturado con HCL 0.1 N. Se agitó la solución por un minuto y posteriormente se trasfirió a un tubo de centrifuga y se separaron las dos fases, por $5 \mathrm{~min}$, a una velocidad de $3000 \mathrm{rpm}$. Se tomó una alícuota de la capa superior con una pipeta y transfirió a una celda de vidrio de cuarzo para el análisis espectrofotométrico. Por último, se midió la absorbancia de los extractos a una longitud de onda de $544 \mathrm{~nm}$. Se empleó una solución de alcohol n-amílico saturado con HCL $0.1 \mathrm{~N}$ como blanco.

Determinación de alcaloides teobromina y cafeína: La determinación de Teobromina y Cafeína, permite establecer la relación Teobromina/Cafeína (T/C), parámetro medible y promisorio para la diferenciación de cacaos por su origen. El método está basado en el Método Oficial 980.14 de la Association of Official Analytical Chemists (1995). En un matraz Erlenmeyer de $250 \mathrm{~mL}$ se pesaron $0.3 \mathrm{~g}$ de muestras desengrasadas, se adicionaron $90 \mathrm{~mL}$ de agua bidestilada y $10 \mathrm{~mL}$ de estándar interno, el matraz con la solución se calentó en una placa por $30 \mathrm{~min}$ hasta que el volumen se redujo a $50 \mathrm{~mL}$, posteriormente el matraz Erlenmeyer fue retirado de la plancha de calentamiento y se le adicionó $1.0 \mathrm{~mL}$ de solución Carrez 1 y Carrez 2, después se filtró el extracto a través de papel Whatman $\mathrm{N}^{\circ} 4$ en un matraz volumétrico de $100 \mathrm{~mL}$, se lavó el filtrado y se aforó con agua bidestilada. Se tomó una alícuota de filtrado, y se hizo pasar por una membrana millipore de $0.22 \mu \mathrm{m}$ y se colocó en un vial para inyección en el HPLC, por último, el extracto restante se almaceno en refrigeración.

Análisis estadístico: Todas las mediciones se realizaron por triplicado de manera independiente, usando un diseño completamente al azar. Los resultados se sometieron a un análisis de varianza y comparación de medias Duncan $(\mathrm{P} \leq 0.05)$. El análisis de datos se realizó con el paquete estadístico SAS Ver 9.0 (SAS, 2001). El análisis se completó con componentes principales y cladístico, comparando las accesiones, con la especie T. mamosum como taxón externo. El cual fue conducido empleando el algoritmo de parsimonia del programa de Nona (Goloboff, 1993) en conjunto con el programa WinClada (Nixon, 2002). El resultado final se obtuvo con las siguientes características: La búsqueda heurística se dirigió con 1000 repeticiones stepwise y TBR ramas combinando con MULTIPARS activado. Todos los caracteres 
fueron valorados igualmente. Se calcularon valores de Bootstrap y Jackknife para los nodos con 1000 repeticiones, 1000 repeticiones de la búsqueda (mult*1000), con TBR combinado y 10000 como número máximo de juego de árboles.

\section{RESULTADOS}

De acuerdo con el análisis de varianza se obtuvieron diferencias significativas $(\mathrm{P}<$ 0.001 ) entre especies para el contenido de fenoles, flavonoides y actividad antioxidante por el método de ABTS, cafeína, teobromina y la relación $\mathrm{T} / \mathrm{C}$, así como, diferencias $(\mathrm{P}<0.05)$ en las variables actividad antioxidante por el método de DDPH y contenido de antocianinas.

Para el grupo de genotipos derivados de T. cacao, el análisis de varianza mostró diferencias significativas $(\mathrm{P}<0.001)$ para la actividad antioxidante por el método de DDPH, contenido de antocianinas y la relación $\mathrm{T} / \mathrm{C}$; así como, diferencias $(\mathrm{P}<0.05)$ para la capacidad antioxidante por el método de ABTS, cafeína y teobromina, resaltando que, para el contenido de fenoles y flavonoides, no hubo diferencia.

La comparación de medias de las variables analizadas en las cuatro especies mostró que $T$. bicolor registró los menores valores, excepto en la relación T/C (Tabla 2; Tabla 3). La tendencia de mayores valores para casi todas las variables fue en lo general para las especies silvestres, lo cual podría deberse a que los metabolitos secundarios evaluados, funcionan en la adaptación y supervivencia ante situaciones de estrés ambiental. Únicamente $T$. cacao, registró los mayores valores para el contenido de teobromina y cafeína (Tabla 3 ).

El mayor contenido de fenoles se observó en T. mammosum. En cuanto a T. cacao, los valores fueron inferiores a los registrados en T. mammosum, pero superiores a $T$. angustifolium y el menor en $T$. bicolor (Tabla 2). Entre genotipos de $T$. cacao, el contenido de fenoles osciló de 7.5 a $85.2 \mathrm{mg} \mathrm{g}^{-1}$, y no hubo tendencia clara entre forasteros, trinitarios y criollos (Tabla 2; Tabla 4).

Para el contenido de flavonoides se observó casi el mismo comportamiento que en fenoles. El mayor contenido lo registró T. mammosum y el menor T. bicolor. No se observó tendencia entre especies silvestres y domesticada; sin embargo, T. cacao mostró valores inferiores respecto a $T$. mammosum, pero mayores que $T$. angustifolium. Los genotipos derivados de $T$. cacao, mostraron un contenido de flavonoides entre 6.57 y $69.6 \mathrm{mg} \mathrm{g}^{-1}$, y no se observó una tendencia clara entre forasteros, trinitarios y criollos (Tabla 2; Tabla 4).

TABLA 2

Comparación de medias del contenido de fenoles, flavonoides, actividad antioxidante (ABTS, DDPH) y antocianinas (ANTO) en genotipos de cuatro especies de Theobroma

TABLE 2

Comparison of means of the content of phenols, flavonoids, antioxidant activity (ABTS, DDPH) and anthocyanins (ANTO) in genotypes of four species of Theobroma

\begin{tabular}{lccccc}
$\quad$ Genotipo & Fenoles & Flavonoides & ABTS & DDPH & ANTO \\
T. cacao Forastero & $33.18 \mathrm{~b}$ & $30.35 \mathrm{ab}$ & $76.78 \mathrm{a}$ & $49.23 \mathrm{~cd}$ & $1.11 \mathrm{a}$ \\
T. cacao Trinitario & $38.78 \mathrm{ab}$ & $35.86 \mathrm{~b}$ & $69.97 \mathrm{a}$ & $49.8 \mathrm{bcd}$ & $0.61 \mathrm{ab}$ \\
T. cacao Criollo & $37.06 \mathrm{a}$ & $33.20 \mathrm{ab}$ & $68.98 \mathrm{a}$ & $51.5 \mathrm{abc}$ & $0.74 \mathrm{ab}$ \\
T. cacao & $36.57 \mathrm{a}$ & $33.26 \mathrm{ab}$ & $71.31 \mathrm{a}$ & $50.44 \mathrm{ab}$ & $0.80 \mathrm{ab}$ \\
T. mammosum & $42.05 \mathrm{a}$ & $42.18 \mathrm{a}$ & $72.06 \mathrm{a}$ & $52.47 \mathrm{ab}$ & $1.11 \mathrm{a}$ \\
T. angustifolium & $30.16 \mathrm{a}$ & $26.74 \mathrm{~b}$ & $63.90 \mathrm{a}$ & $53.15 \mathrm{a}$ & $0.66 \mathrm{ab}$ \\
T. bicolor & $3.37 \mathrm{c}$ & $1.61 \mathrm{c}$ & $6.54 \mathrm{~b}$ & $48.10 \mathrm{~d}$ & $0.02 \mathrm{~b}$ \\
\hline
\end{tabular}

Letras diferentes en la misma columna son diferentes estadísticamente.

Different letters in the same column are statistically different. 
TABLA 3

Comparación de medias del contenido de cafeína, teobromina y su relación (T/C) en genotipos de cuatro especies de Theobroma

TABLE 3

Comparison of means of the content of caffeine, theobromine and their relationship (T/C) in genotypes of four species of Theobroma

\begin{tabular}{lccc}
$\quad$ Genotipo & Cafeína $\left(\mathrm{mg} \mathrm{g}^{-1}\right.$ peso seco) & Teobromina $\left(\mathrm{mg} \mathrm{g}^{-1}\right.$ peso seco) & T/C \\
T. cacao Forastero & $3.90 \mathrm{~b}$ & $5.87 \mathrm{a}$ & $1.52 \mathrm{c}$ \\
T. cacao Trinitario & $4.48 \mathrm{a}$ & $6.69 \mathrm{a}$ & $1.50 \mathrm{c}$ \\
T. cacao Criollo & $4.47 \mathrm{a}$ & $6.68 \mathrm{a}$ & $1.49 \mathrm{c}$ \\
T. cacao & $4.32 \mathrm{a}$ & $6.47 \mathrm{a}$ & $1.50 \mathrm{c}$ \\
T. mammosum & $3.44 \mathrm{~b}$ & $5.24 \mathrm{a}$ & $1.52 \mathrm{c}$ \\
T. angustifoliun & $2.10 \mathrm{~b}$ & $3.34 \mathrm{~b}$ & $1.59 \mathrm{~b}$ \\
T. bicolor & $0.32 \mathrm{c}$ & $0.84 \mathrm{c}$ & $2.61 \mathrm{a}$ \\
\hline
\end{tabular}

T/C: Relación teobromina/cafeína. Letras diferentes en la misma columna son diferentes estadísticamente.

$\mathrm{T} / \mathrm{C}$ : Theobromine/caffeine ratio. Different letters in the same column are statistically different.

TABLA 4

Comparación de medias del contenido de fenoles, flavonoides, actividad antioxidante (ABTS y DDPH) en genotipos de cuatro especies de Theobroma

TABLE 4

Comparison of means of the content of phenols, flavonoids, antioxidant activity (ABTS and DDPH) in genotypes of four species of Theobroma

\begin{tabular}{|c|c|c|c|c|c|c|c|}
\hline \multicolumn{2}{|c|}{ Fenoles } & \multicolumn{2}{|c|}{ Flavonoides } & \multicolumn{2}{|c|}{ ABTS } & \multicolumn{2}{|c|}{ DDPH } \\
\hline Genotipo & Media & Genotipo & Media & Genotipo & Media & Genotipo & Media \\
\hline $\mathrm{C} 22$ & $85.2 \mathrm{a}$ & $\mathrm{C} 22$ & $69.6 \mathrm{a}$ & $\mathrm{C} 4$ & $86.1 \mathrm{a}$ & $\mathrm{C} 21$ & $53.3 \mathrm{a}$ \\
\hline F13 & $48.3 \mathrm{~b}$ & F11 & $47.9 \mathrm{~b}$ & $\mathrm{C} 2$ & $85.4 \mathrm{a}$ & C6 & $53.3 \mathrm{a}$ \\
\hline T12 & $48.0 \mathrm{~b}$ & $\mathrm{~T} 1$ & $44.9 \mathrm{bc}$ & F11 & $85.2 \mathrm{a}$ & $\mathrm{C} 22$ & $52.8 \mathrm{ab}$ \\
\hline F5 & 37.4 fghi & $\mathrm{T} 4$ & $34.8 \mathrm{jklmni}$ & T11 & $73.8 \mathrm{ijkl}$ & $\mathrm{C} 20$ & $51.0 \mathrm{jkl}$ \\
\hline C6 & 37.3 fghil & F5 & $34.1 \mathrm{jklmn}$ & $\mathrm{T} 12$ & $73.3 \mathrm{jklm}$ & $\mathrm{C} 2$ & $51.0 \mathrm{jkl}$ \\
\hline $\mathrm{C} 21$ & 37.2 ghil & $\mathrm{C} 21$ & $34.0 \mathrm{jklmn}$ & C9 & $73.2 \mathrm{jklm}$ & T14 & $50.7 \mathrm{klm}$ \\
\hline $\mathrm{C} 1$ & 16.37 op & $\mathrm{C} 1$ & $13.5 \mathrm{uv}$ & $\mathrm{C} 16$ & $42.7 \mathrm{v}$ & F11 & $46.2 \mathrm{t}$ \\
\hline $\mathrm{F} 3$ & $11.3 \mathrm{qp}$ & F3 & $8.83 \mathrm{vw}$ & $\mathrm{C} 20$ & $35.1 \mathrm{w}$ & $\mathrm{T} 12$ & $42.0 \mathrm{u}$ \\
\hline $\mathrm{C} 18$ & $7.5 \mathrm{q}$ & C18 & $6.57 \mathrm{w}$ & $\mathrm{C} 18$ & $17.3 \mathrm{x}$ & F3 & $40.0 \mathrm{v}$ \\
\hline
\end{tabular}

Letras distintas en la misma columna son diferentes estadísticamente.

Different letters in the same column are statistically different.

Con relación a la capacidad de inhibir el radical libre 2,2-azinobis (3-etilbenzotiazolino6-ácidosulfonico) (ABTS); no se observaron diferencias estadísticas entre especies, excepto en $T$. bicolor quien registró el menor valor. Los genotipos derivados de $T$. cacao mostraron variación de 17.3 a 86.1.

En cuanto a la capacidad de inhibir el radical libre 1,1-difenil-2-picrilhidrazil (DPPH), se observó lo contrario que en ABTS, ya que la mayor actividad antioxidante fue vista en $T$. angustifolium, mientras que T. bicolor siguió mostrando el valor menor. Respecto a los genotipos derivados de T. cacao, los valores más altos fueron obtenidos en los tipos criollos (53.3 a 40) (Tabla 2; Tabla 4). El contenido de antocianinas osciló de 0.01 a $3 \mathrm{mg} \mathrm{g}^{-1}$ de peso seco, y el mayor contenido fue para genotipos 
derivados de T. cacao (forastero), seguido de T. mammosum y el menor valor en $T$. bicolor.

Para la variable cafeína (1.8 a $6.7 \mathrm{mg} \mathrm{g}^{-1}$ peso seco) el valor más alto se observó en genotipos derivados de T. cacao (trinitarios y criollos), mientras que, entre especies, el menor valor fue en T. bicolor (Tabla 2; Tabla 5). Lo mismo se presentó con los valores de teobromina (2.9 a $9.8 \mathrm{mg} \mathrm{g}^{-1}$ peso seco), y en cuanto a la relación T/C (1.62 a 1.46), la especie T. bicolor registró el mayor valor, y para los genotipos domesticados de T. cacao, el tipo forastero fue el más relevante (Tabla 3; Tabla 5).

Se observó una correlación positiva entre el contenido de flavonoides y fenoles con la actividad antioxidante de las especies de Theobroma spp. y los genotipos de T. cacao (Fig. $1)$; lo anterior sugiere que conforme incrementa el contenido de fenoles y flavonoides en las semillas de cacao es mayor la capacidad antioxidante.

El análisis de componentes principales sugiere que en el biplot de variables del primer componente (CP1) separa al atributo del contenido de fenoles, flavonoides, cafeína y teobromina, el cual representó el 59.5 \% (Fig. 2). Así mismo, el atributo de la actividad antioxidante
(DDPH) y contenido de antocianinas de las semillas de cacao representó el $15.7 \%$ de la variabilidad del segundo componente (CP2), y en general, ambos componentes representaron $75.3 \%$ de la variabilidad total. En la Fig. 2 se puede apreciar que la mayoría de los genotipos con mayor actividad antioxidante (DDPH) y de alcaloides (teobromina y cafeína) fueron los criollos, seguidos de trinitarios (T. cacao).

El análisis de conglomerados de los genotipos evaluados diferenció cinco grupos (Fig. $3)$. El grupo 1 representó $56.6 \%$ (F1, 6, 5, 8 y $13 ; \mathrm{T} 1,2,3,5,6,7,8,9,10$ y $14 ; \mathrm{C} 2,3,5,6,7$, $8,9,10,11,12,13,17,19$ у $21 ;$ T. mammosum). Este grupo derivó dos subgrupos caracterizados por mayor cantidad de genotipos criollos ( $T$. cacao) con fenoles (40.76), flavonoides (37.45) actividad antioxidante ABTS (76.06) y DDPH (50.97), así como, antocianinas (0.68), cafeína (4.82) y teobromina (7.717).

El grupo 2 representó $11.3 \%$ del total evaluado (F 11, 12 y 14; T 11; C4 y 22), y fue el de mayor contenido de fenoles (53.31), flavonoides (48.2) y ABTS (81.1), valor intermedio en DDPH (47.67) y mayor valor en antocianinas (2.12).

TABLA 5

Comparación de medias del contenido de antocianinas, cafeína, teobromina y relación teobromina/cafeína (T/C) en genotipos de cuatro especies de Theobroma

TABLE 5

Comparison of means of the content of anthocyanins, caffeine, theobromine and the theobromine/caffeine ratio (T/C) in genotypes of four species of Theobroma

\begin{tabular}{|c|c|c|c|c|c|c|c|}
\hline \multicolumn{2}{|c|}{ Antocianinas } & \multicolumn{2}{|c|}{ Cafeína (C) } & \multicolumn{2}{|c|}{ Teobromina $(\mathrm{T})$} & \multicolumn{2}{|c|}{ Relación T/C } \\
\hline Gen & Media & Gen & Media & Gen & Media & Gen & Media \\
\hline F11 & $3.0 \mathrm{a}$ & $\mathrm{C} 2$ & $6.7 \mathrm{a}$ & $\mathrm{C} 2$ & $9.8 \mathrm{a}$ & F3 & $1.62 \mathrm{a}$ \\
\hline $\mathrm{C} 4$ & $2.4 \mathrm{~b}$ & F8 & $6.6 \mathrm{a}$ & F8 & $9.7 \mathrm{a}$ & F9 & $1.61 \mathrm{a}$ \\
\hline $\mathrm{C} 22$ & $2.3 \mathrm{~b}$ & C11 & $6.2 \mathrm{~b}$ & C11 & $9.1 \mathrm{~b}$ & $\mathrm{C} 20$ & $1.57 \mathrm{~b}$ \\
\hline F7 & 0.57 lmno & $\mathrm{T} 11$ & $4.5 \mathrm{o}$ & $\mathrm{T} 11$ & 6.8 o & T8 & $1.49 \mathrm{ijk}$ \\
\hline $\mathrm{T} 7$ & 0.56 lmno & F6 & $4.4 \mathrm{p}$ & F6 & $6.6 \mathrm{p}$ & $\mathrm{C} 13$ & $1.49 \mathrm{ijk}$ \\
\hline $\mathrm{T} 5$ & 0.56 lmno & T9 & $4.3 \mathrm{p}$ & T9 & $6.5 \mathrm{p}$ & $\mathrm{T} 10$ & $1.49 \mathrm{ijk}$ \\
\hline $\mathrm{C} 21$ & 0.04 qrs & $\mathrm{C} 20$ & $2.3 \mathrm{z}$ & $\mathrm{C} 20$ & $3.6 \mathrm{z}$ & $\mathrm{T} 2$ & $1.47 \mathrm{mn}$ \\
\hline $\mathrm{C} 12$ & $0.02 \mathrm{rs}$ & F9 & $1.9 \mathrm{z}$ & F9 & $3.0 \mathrm{z}$ & $\mathrm{C} 2$ & $1.46 \mathrm{n}$ \\
\hline $\mathrm{C} 10$ & $0.01 \mathrm{~s}$ & F3 & $1.8 \mathrm{z}$ & F3 & $2.9 \mathrm{z}$ & F8 & $1.46 \mathrm{n}$ \\
\hline
\end{tabular}

T/C: Relación teobromina/cafeína. Letras distintas en la misma columna son diferentes estadísticamente.

$\mathrm{T} / \mathrm{C}$ : Theobromine/caffeine ratio. Different letters in the same column are statistically different. 

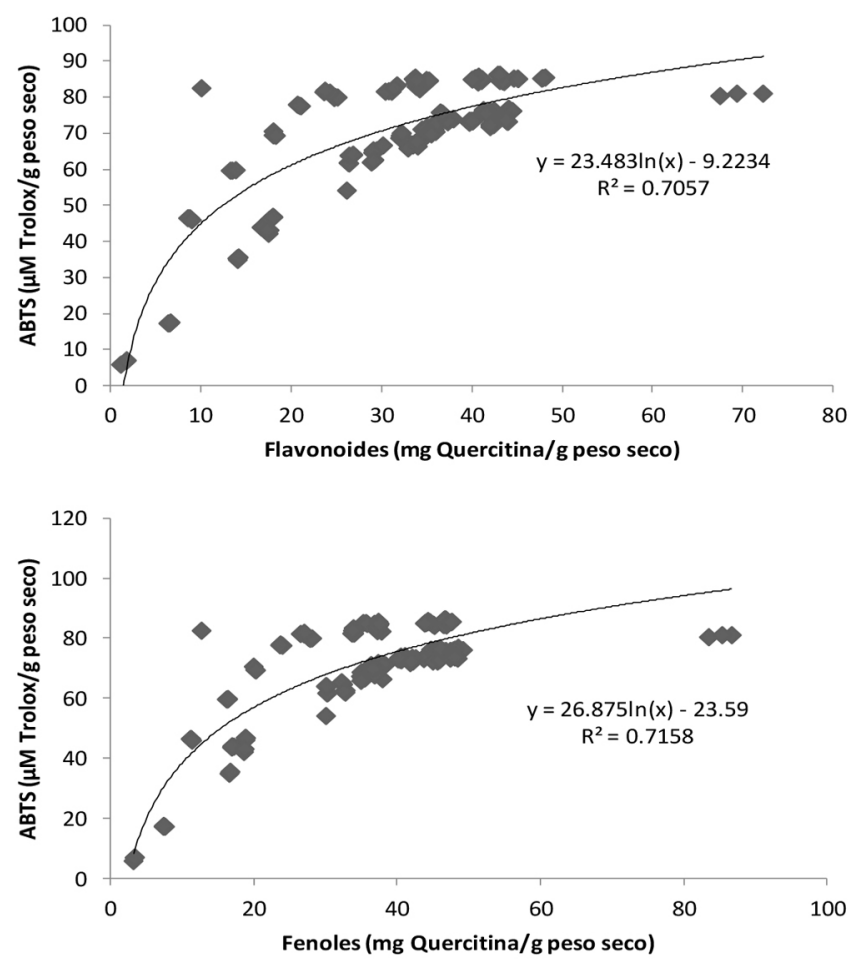

Fig. 1. Correlación entre el contenido de flavonoides, fenoles y la capacidad antioxidante de acuerdo con el método ABTS de genotipos de cuatro especies de Theobroma.

Fig. 1. Correlation between the content of flavonoids, phenols and antioxidant capacity according to the ABTS method of genotypes of four species of Theobroma.

El grupo 3 represento $24.5 \%$ (F 2, 4, 7, 9 y $10 ;$ T 4,12 у $13 ; C 1,14,15$ y $16 ;$ T. anfustifolium), caracterizado por presentar valores intermedios en todas las variables, mientras que el grupo 4 significó el $5.6 \%$ (F 3; C 18 y 20) que al igual que el grupo 5 (1.9\% . bicolor $)$, tuvieron los valores más bajos en todas las variables.

Análisis cladístico: El análisis cladístico generó un árbol de consenso estricto $(\mathrm{L}=137$, $\mathrm{Ci}=37, \mathrm{Ri}=76$ ) (Fig. 4), formando dos grupos, el primero, sustentado por los caracteres 1 y 2 (fenoles y flavonoides), y el segundo por los caracteres 3, 6 y 7 (ABTS, CAF y TEO). El primer clado agrupó 16 genotipos, en el cual los caracteres 1 (fenoles), 3 (ABTS), 4 (DDPH), 6 (cafeína) y 7 (teobromina), fueron las novedades (caracteres apomórficos). En este grupo el carácter 1 (fenoles) y 7 (teobromina) agruparon a $T$. angustifolium y al genotipo F9 como taxones hermanados, de los cuales no se pudieron recuperar caracteres sinapomórficos, no obstante, su hermandad se sustenta en el carácter 4 (DDPH) y 7 (teobromina) como carácter hipoplásico y sinapomórfico, respectivamente. El subgrupo 2 se sustentó por la homoplasia representada por el carácter 3 (ABTS), que muestra la hermandad de los taxones F2, T13 y C9, teniendo como novedad a la cafeína, antocianinas y teobromina, respectivamente.

El carácter fenoles (1) permitió la distinción del subgrupo que incorporó a ocho genotipos (C20, C15, C16, T4, C1, C18, T. bicolor, y F3). En este grupo los caracteres 1 y 2 (fenoles y flavonoides) distinguieron a los materiales C18, T. bicolor, y F3. Los caracteres 6, 7 y 8 (cafeína, teobromina y relación $\mathrm{T} / \mathrm{C}$ ) resultaron como novedades de la hermandad entre 


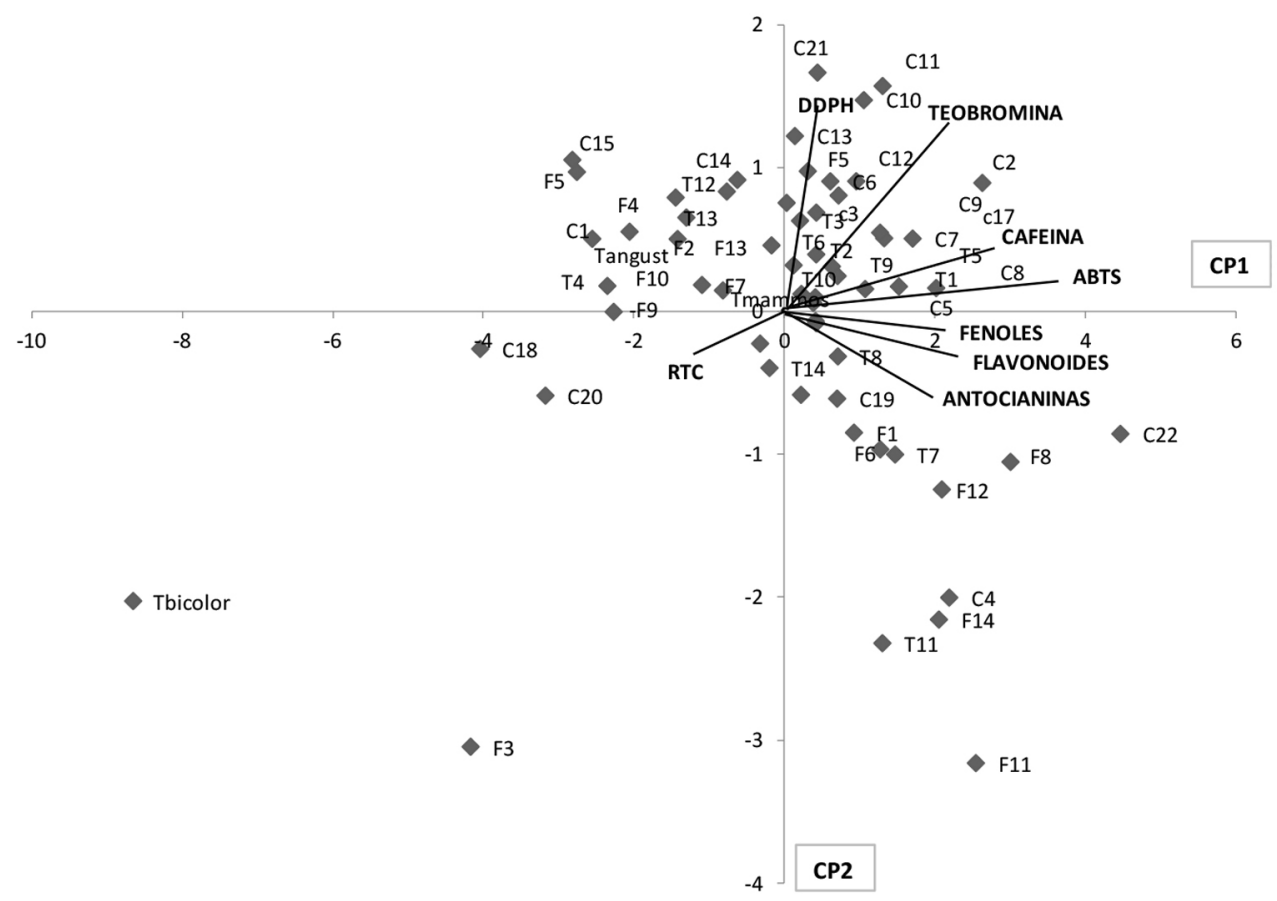

Fig. 2. Dispersión de genotipos de cuatro especies de Theobroma con base en ocho variables bioquímicas de semilla.

Fig. 2. Genotype dispersion of four Theobroma species based on eight biochemical seed variables.

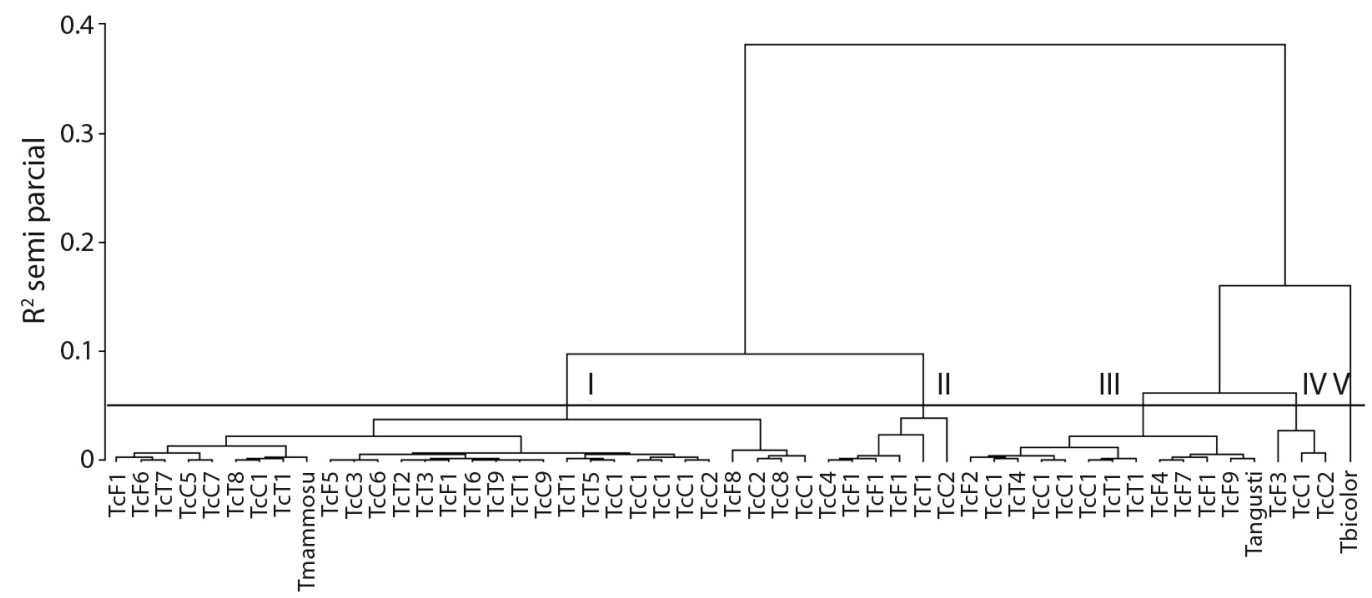

Genotipo

Fig. 3. Dendograma de genotipos de cuatro especies de Theobroma con base en ocho variables bioquímicas de semilla.

Fig. 3. Genotype dendogram of four Theobroma species based on eight biochemical seed variables. 


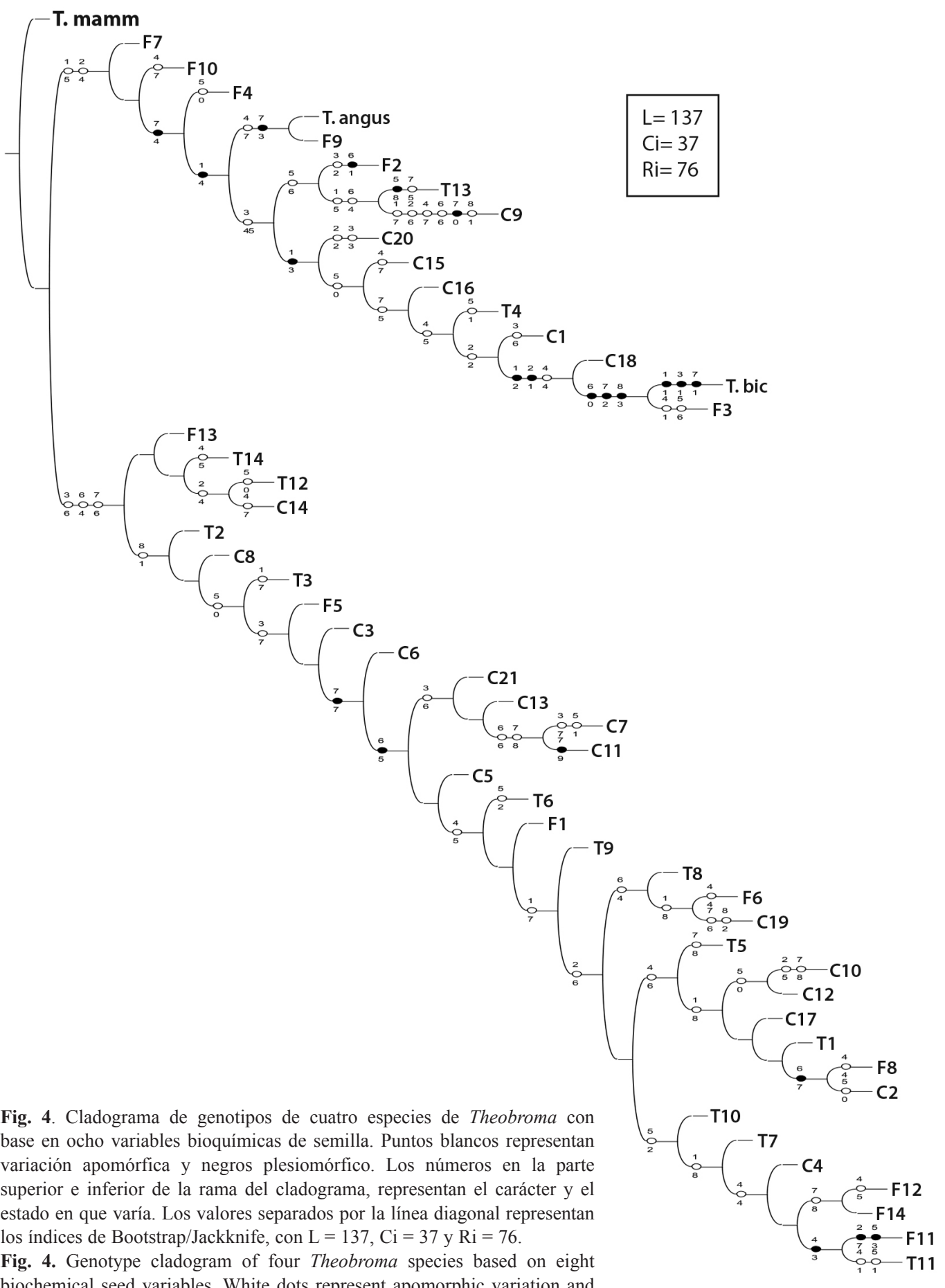
biochemical seed variables. White dots represent apomorphic variation and black plesiomorphic. The numbers at the top and bottom of the cladogram branch represent the character and the state in which it varies. The values separated by the diagonal line represent the Bootstrap/Jackknife indices, with $\mathrm{L}=137, \mathrm{Ci}=37$ and $\mathrm{Ri}=76$. 
T. bicolor y F3, mientras que, para T. bicolor, las novedades fueron la cantidad de fenoles, ABTS y teobromina, y para F3 como caracteres heredados del ancestro común, la cantidad de fenoles y teobromina.

Los caracteres sinapomórficos (compartidos) 3, 6, 7 (ABTS, cafeína y teobromina) permitieron la separación del segundo subclado en cinco subgrupos (Fig. 4), de los cuales, en el primero de ellos no fue posible recuperar caracteres de distinción agrupando a los genotipos F3, T14, T12 y C14. La subdivisión de los restantes cuatro subclados se sustentó por el carácter 7 (teobromina) como una novedad en el grupo, posteriormente el carácter cafeína (6) de igual forma como novedad dio la separación de los cuatro subclados. El primer subclado agrupó a los genotipos (taxones) C21, C13, C7 y $\mathrm{C} 11$ y fueron sinapomórficos, compartiendo los caracteres 6 y 7, y aunque no fue posible recuperar caracteres sinapomórficos para C21, se apreció la hermandad de C7 y C11, destacando $\mathrm{C} 11$ por su concentración de teobromina como novedoso.

El subclado dos, agrupo a los taxones T8, F6 y C19, resaltando la hermandad de F6 y C19, cuya novedad fue el carácter 4 (DDPH), mientras que para $\mathrm{F} 6$ fue la concentración de teobromina, y la relación $\mathrm{T} / \mathrm{C}$ para $\mathrm{C} 19$. El clado integrado por T8, F6 y C19 sustentado por el carácter cafeína (6) como novedad del grupo, T8 no presentó caracteres sinapomórficos, mientras que la novedad en F6 fue el DDPH (4), en C19 las novedades fueron los caracteres 7 y 8 (teobromina, y la relación T/C).

La capacidad antioxidante (DDPH) fue el carácter que distinguió al tercer subclado agrupando los genotipos T5, C10, T1, C12, C17, F8 y $\mathrm{C} 2$, de los cuales $\mathrm{C} 10$ y C12 estuvieron hermanados, al igual que F8 y C2. Para T1 y C17 no se identificaron caracteres sinapomórficos, mientras que $\mathrm{C} 10$ y $\mathrm{C} 12$ se hermanaron por el carácter antocianinas, destacando el carácter flavonoides (2), y para C10 la teobromina (7). La hermandad de F8 y C2 se sustentó por el carácter (cafeína, 6), destacando como novedad en F8 el carácter DDPH (4) y para C2 a las antocianinas (5).
Las antocianinas (5) soportaron la formación del subclado mas evolucionado del presente estudio, y se formó por los genotipos T10, T7, C4, F2, F14, F11 y T11, de los cuales F2 y F4 se hermanaron, al igual que F11 y T11. Para los genotipos T10, T7, C4, no se pudieron recuperar caracteres sinapomórficos; sin embargo, para el resto de los genotipos el carácter DDPH (4) sustentó la agrupación de taxones hermanos.

Para el caso de F2 y F14 el carácter teobromina (7) sustentó la hermandad como un carácter hipoplásico o cambio evolutivo, mientras que para F11 y T11 el carácter DDPH (4) fue un carácter sinapomórfico. Para F2, la sinapomorfía fue el DDPH. En la hermandad de los taxones F11 y T11, la novedad evolutiva fue el carácter DDPH (4), ya que F11 presentó como novedades evolutivas los caracteres ABTS y antocianinas $(2,5)$, mientras que las sinapomorfías de T11 fueron DDPH y antocianinas. En F14 no se determinaron sinapomorfías.

\section{DISCUSIÓN}

Es importante señalar que el grado de diferenciación de cada especie es distinta, las especies consideradas como silvestres son $T$. mammosun y $T$. angustifoliun; mientras que la semi domesticada para México es T. bicolor, y como especie domesticada T. cacao (Rondón \& Cumana, 2005; Sousa-Silva \& Figueira, 2005; McCoy et al., 2019). La correlación directa entre el contenido de flavonoides y fenoles con la actividad antioxidante en las especies de Theobroma, y los genotipos derivados de T. cacao sugiere que conforme incrementa el contenido de fenoles y flavonoides en las semillas de cacao, aumenta la actividad antioxidante. Perea, Cadena, y Herrera (2009), reportan una relación semejante, y sugieren que la cantidad de polifenoles y la actividad antioxidante parecen depender del contenido de sólidos no grasos presentes en los productos finales. El contenido de polifenoles totales se correlaciona con la actividad antioxidante expresada como poder reductor, presentando coeficientes de correlación $\mathrm{r}^{2}$ de 0.768 y 0.734 
respectivamente (Padilla et al., 2008), lo cual sugiere que, a mayor contenido de polifenoles, mayor actividad antioxidante. Lo anterior, es semejante a lo registrado en esta investigación. Estos resultados difieren a los indicados por Ramírez et al. (2013) quienes reportaron una correlación inversa entre contenido de fenoles totales y actividad antioxidante en semillas de genotipos de cacao de Chiapas, México.

Se observó una amplia variación en el contenido de polifenoles en las especies de Theobroma evaluadas, y dentro de los genotipos derivados de T. cacao, que puede deberse en gran medida a la disponibilidad de nutrientes del suelo donde se han desarrollado, ya que el tipo de suelo tiene gran influencia en la acumulación de polifenoles como lo sugieren Waśkiewicz, Muzolf-Panek, y Goliński (2013), quienes mencionan que el contenido fenólico en las plantas y su actividad antioxidante dependen de factores biológicos (genotipo, órgano y ontogenia), así como de las condiciones edáficas y ambientales (temperatura, salinidad, estrés hídrico e intensidad de la luz).

Los autores Karim, Azlan, Ismail, Hashim, y Abdullah (2014), mencionan que las actividades antioxidantes de los extractos en cacao fueron aportadas por varios compuestos bioactivos que actúan sinérgicamente. Adamson et al. (1999), indican que las diferencias en la cantidad de polifenoles, puede modificarse por el método de beneficiado, el cual puede causar alteraciones químicas en el contenido de antioxidantes. Durante el secado el aire penetra al grano a través de la testa, oxidando a los polifenoles, provocando reacciones bioquímicas internas que contribuyen a disminuir la astringencia, por lo que condicionan en gran parte el sabor del producto (Álvarez et al., 2010).

Estudios realizados por Lattanzio, Lattanzio, y Cardinali (2006), mencionan que las plantas necesitan compuestos fenólicos para la pigmentación, crecimiento, reproducción, resistencia a patógenos y otras funciones. Por lo tanto, representan mecanismos adaptativos en la selección natural durante la evolución. Los compuestos fenólicos en los cacaos son metabolitos secundarios (Ms) involucrados en su defensa contra hongos patógenos y herbivoría, y pueden variar dependiendo del órgano que se trate (Quiñones, Trujillo, Capdesuñer, Quirós, \& Hernández, 2013), por ejemplo, $T$. mammosum, cuyo estatus silvestre le obliga a mantener mayor contenido de fenoles y flavonoides. DiFerdinando, Brunetti, Agati, yTattin (2014), mencionan que los polifenoles son probablemente los Ms de mayor versatilidad biogénica, lo cual permite a las plantas responder rápidamente a agentes de estrés impredecibles de diferente origen, como a los que están sometidos las especies silvestres de Theobroma .

Reportes de Pourcel, Routaboul, Cheynier, Lepiniec, y Debeaujon (2007), indican que muchas de las funciones biológicas de los flavonoides se atribuyen a su potencial citotóxico y actividad antioxidante. La oxidación de flavonoides contribuye a estas propiedades químicas y biológicas y puede conducir a la formación de pigmentos marrones en los tejidos vegetales, así como a los alimentos y bebidas derivados de las plantas. Estos mismos autores mencionan que la oxidación de flavonoides en planta es catalizada principalmente por polifenol oxidasas (catecol oxidasas y lacasas) y peroxidasas que son inducidas durante el desarrollo de semillas y plantas, como respuesta a presiones ambientales, tales como los ataques de patógenos. A este respecto, tantos las especies silvestres de Theobroma, como los genotipos derivados de $T$. cacao y principalmente el grupo de los criollos son muy atacados por diferentes organismos plaga durante su cultivo (Hernández-Gómez et al., 2015).

Desde el punto de vista de la salud humana, autores como Abbe y Amin (2008) y de Rezende Mudenuti et al. (2018) mencionan la relación entre la acción antioxidante del cacao y la salud, sugiriendo su declaración como producto saludable debido al contenido de flavonoides. De acuerdo con Hii et al. (2009), los polifenoles en las semillas de cacao contribuyen con 12 a $18 \%$ de su peso seco, y se pueden distinguir tres grupos principales: las catequinas $(37 \%)$, las antocianinas (4\%) y las proantocianidinas (58 \%). La catequina principal es (-) - epicatequina con hasta $35 \%$ 
de contenido de polifenoles, y el procesamiento de las semillas como la fermentación (RiveraFernández et al., 2012), secado, tostado y molido, podrían influir en los polifenoles presentes y sus productos derivados como lo reportan Chávez y Ordoñez (2013). De acuerdo a Orfa, Ordoñez, Mandujano, y Arévalo (2013), las diferentes formas de catequinas, ya sean $(+)$ o (-) se absorben de manera diferente, y dependen también de las condiciones ambientales y grupo genético al que pertenezca la variedad de cacao, por ejemplo, forastero, trinitario o criollo (Fig. 4).

de Rezende Mudenuti et al. (2018) compararon el contenido de fenoles y alcaloides en chocolates elaborados con granos tostados y sin tostar, registrando que la actividad antioxidante fue mayor en granos $\sin$ tostar $(\geq 50 \%)$ debido al contenido de metabolitos solubles que en las fórmulas tradicionales de chocolate a base de grano tostado. Estudios realizados en nueces de barú (Dipteryx alata Vog) con y sin cáscara, mostraron que el tostado no redujo significativamente el contenido fenólico total en comparación con la nuez cruda (Bonilla-Lemos, de Almeida, Fernandes, \& Zambiazi, 2012).

Dentro de los genotipos derivados de $T$. cacao, se observó que los tipos criollos registraron menor cantidad de antocianinas comparado con el tipo forastero; lo cual podría estar relacionado con el color de los cotiledones, ya que, en los primeros, la semilla es de color blanco, mientras que, en los segundos, la coloración es violácea atribuida a las antocianinas.

La variación encontrada en las especies de Theobroma, y los derivados de T. cacao de acuerdo con el análisis de componentes principales estuvo dado por el contenido de fenoles, flavonoides, cafeína, teobromina y actividad antioxidante. Resultados similares se han reportado en la cereza (Cerasus humilis) en China (Wang et al., 2018) donde se ha aprovechado este diferencial en el contenido de fenoles y actividad antioxidante en actividades de selección de variedades. Lo mismo se ha encontrado en esta investigación al observar genotipos agrupados por mayor contenido de fenoles y actividad antioxidante lo cual podría ser aprovechado en selección varietal por mayor contenido de estos metabolitos. Othman et al. (2007), reportaron variación y diferencias en la actividad antioxidante y contenido fenólico total de semillas de cacao de diferentes países (Malasia, Ghana, Costa de Marfil y Sulawesi). Respecto al contenido de teobromina y cafeína, al igual que las otras variables se encontró una amplia variación, lo cual podría explicar al origen genético de los genotipos y el nivel de domesticación de las especies (Waśkiewicz et al., 2013). Aun cuando el contenido de cafeína y teobromina fue mayor en el grupo de cacaos criollos, la relación $\mathrm{T} / \mathrm{C}$ no permitió distinguir entre grupos genéticos de $T$. cacao como lo reportaron Espin, Samaniego, Harue, y Jimenez (2007).

La variación encontrada en el contenido de fenoles, flavonoides, actividad antioxidante, antocianinas, teobromina y cafeína sugiere un efecto por el grado de domesticación de las especies evaluadas, mientras que en el grupo de genotipos derivados de T. cacao (forastero, trinitario y criollo) fue por el origen de las semillas. Se observó que el grado de domesticación de las especies influye en el contenido de fenoles y actividad antioxidante. Se distinguieron relaciones filogenéticas entre especies y genotipos evaluados. Los resultados sugieren que las variables bioquímicas evaluadas pueden ayudar a formar criterios para el mejoramiento genético en el complejo derivado de $T$. cacao orientado a la selección de mayor contenido de fenoles y mayor actividad antioxidante.

Declaración de ética: los autores declaran que todos están de acuerdo con esta publicación y que han hecho aportes que justifican su autoría; que no hay conflicto de interés de ningún tipo; y que han cumplido con todos los requisitos y procedimientos éticos y legales pertinentes. Todas las fuentes de financiamiento se detallan plena y claramente en la sección de agradecimientos. El respectivo documento legal firmado se encuentra en los archivos de la revista. 


\section{AGRADECIMIENTOS}

Al Fondo Sectorial SADER-CONACYT México por el financiado del proyecto No. 2017-2-291417.

\section{RESUMEN}

Introducción: Las cualidades organolépticas de las especies aromáticas y sus productos derivados se relacionan directamente con algunas características del sabor, color y valor nutricional y dependen en gran medida de su origen genético y contenido de metabolitos secundarios. Objetivo: se evaluó la actividad antioxidante de diferentes genotipos de Theobroma spp. de México, con el fin de distinguir cualidades promisorias para el mejoramiento genético, y diferenciar rasgos filogenéticos, considerando variables bioquímicas. Métodos: se determinó la cantidad de fenoles, flavonoides y actividad antioxidante mediante ABTS y DDPH, además de contenido de antocianinas, teobromina y cafeína en cuatro especies de Theobroma L., y 50 genotipos derivados de $T$. cacao. Resultados: los resultados fueron analizados mediante un análisis de varianza, prueba de medias, análisis de componentes principales y análisis cladístico. Existen diferencias altamente significativas entre genotipos. El contenido de fenoles varió de 7.5-85 $\mathrm{mg} \mathrm{g}^{-1}$; flavonoides 6.57-69.6- $\mathrm{mg} \mathrm{g}^{-1}$, actividad antioxidante por ABTS de 17.3-86.1 y por DDPH de 40.0-53.3; el contenido de antocianinas de 0.01-3, cafeína de 1.8-6.7 y teobromina de $2.9-9.8 \mathrm{mg} \mathrm{g}^{-1}$. El análisis de componentes principales y cladístico ayudó a explicar la variación encontrada y distinguir caracteres evolutivos y hermandades filogenéticas. La variación en contenido de fenoles, flavonoides, actividad antioxidante, antocianinas, teobromina y cafeína estuvo dada principalmente por el grado de domesticación, mientras que para el grupo de genotipos derivados de $T$. cacao (forastero, trinitario y criollo) fue el origen de las semillas. Conclusión: el grado de domesticación influye en el contenido de fenoles y actividad antioxidante. Los resultados sugieren que las variables evaluadas pueden ayudar a formar criterios para el mejoramiento genético en el complejo derivado de $T$. cacao orientado a la selección de mayor contenido de fenoles y mayor actividad antioxidante.

Palabras clave: fenoles; teobromina; forastero; trinitario; criollo.

\section{REFERENCIAS}

Abbe, M.M., \& Amin, I. (2008). Polyphenols in Cocoa and Cocoa Products: Is There a Link between Antioxidant Properties and Health? Molecules, 13, 2190-2219. DOI: $10.3390 /$ molecules 13092190
Álvarez, C., Tovar, L., García, H., Morillo, F., Sánchez, P., Girón, C., \& De Farías, A. (2010). Evaluación de la calidad comercial del grano de cacao (Theobroma cacao L.) usando dos tipos de fermentadores. Revista Cientifica UDO Agrícola, 10(1), 76-87.

Adamson, G., Lazarus, S., Mitchell, L., Prior, G., Cao, C., \& Jacobs, P. (1999). HPLC method for quantification of procyanidins in cocoa and chocolate samples and correlation to total antioxidant capacity. Journal of Agricultural and Food Chemistry, 47, 4184-4188.

Afoakwa, E. (2010). Chocolate Science and Technology. New Delhi, India: John Wiley \& Sons.

Association of Official Analytical Chemists (1995). Official method 980.14. Theobromine and caffeine in cacao products Liquid Chromatographic method. Arlington, USA: Association of Official Analytical Chemists.

Avendaño-Arrazate, C.H., Martínez-Bolaños, M., \& Mendoza-López, A. (2018). The Cocoa (Theobroma cacao L.) native in Mexico. Biodiversity International Journal, 2(6), 535-536. DOI: 10.15406/ bij.2018.02.00109

Bonilla-Lemos, M.R., de Almeida, E.M., Fernandes, S., \& Zambiazi, R.C. (2012). The effect of roasting on the phenolic compounds and antioxidant potential of baru nuts (Dipteryx alata Vog.). Food Research International, 48(2), 592-597. DOI: $10.1016 / \mathrm{j}$. foodres.2012.05.027

Brand-Williams, W., Cuvelier, M.E., \& Berset, C. (1995). Use of a free radical method to evaluate antioxidant activity. LWT - Food Science and Technology, 28(1), 25-30.

Cádiz-Gurrea, M.L., Lozano-Sanchez, J., Contreras-Gámeza, M., Legeai-Mallet, L., Fernández-Arroyo, S., \& Segura-Carretero, A. (2014). Isolation, comprehensive characterization and antioxidant activities of Theobroma cacao extract. Journal of Functional Foods, 10, 485-498. DOI: 10.1016/j.jff.2014.07.016

Chávez, R.R.E., \& Ordoñez, G.E.S. (2013). Polifenoles Totales, Antocianinas y Capacidad Antioxidante (DPPH y ABTS) durante el procesamiento del licor y polvo de cacao. Revista ECIPerú, 10(1), 43-51. DOI: 10.33017/RevECIPeru2013.0006

Crozier, S., Preston, A., Hurst, J., Payne, M., Mann, J., \& Hainly, L. (2011). Cacao seeds are a "Super Fruit": A comparative analysis of various fruit powders and products. Chemistry Central Journal, 5(1), 1-8. DOI: 10.1186/1752-153X-5-5

de Rezende Mudenuti, N.V., de Camargo, A.C., Shahidi, F., Madeira, T.B., Hirooka, E.Y., \& Grossmann, M.V.E. (2018). Soluble and insoluble-bound fractions of phenolics and alkaloids and their antioxidant activities in raw and traditional chocolate: A comparative study. 
Journal of Functional Foods, 50, 164-171. DOI: 10.1016/j.jff.2018.10.003

DiFerdinando, M., Brunetti, C., Agati, G., \& Tattin, M. (2014). Multiple functions of polyphenols in plants inhabiting unfavorable Mediterranean areas. Environmental and Experimental Botany, 103, 107-116. DOI: 10.1016/j.envexpbot.2013.09.012

Espin, S., Samaniego, I., Harue, W., \& Jimenez, J.C. (2007). La relación teobromina/cafeína asociada a la calidad del cacao Ecuatoriano. Alimentos Ciencia e Ingeniería, 16(2),107-109.

Fisher, N.D.L., Hughes, M., Gerhard-Herman, M., \& Hollenberg, N.K. (2003). Flavanol-rich cocoa induces nitric-oxide-dependent vasodilation in healthy humans. Journal of Hypertension, 21(12), 2281-2286.

Gálvez-Marroquín, L.A., Reyes-Reyes, A.L., AvendañoArrazate, C.H., Hernández-Gómez, E., MendozaLópez, A., \& Díaz-Fuentes, V.H. (2016). Pataxte (Theobroma bicolor Humb. Bonpl) especie subutilizada en México. Agroproductividad, 9(1), 41-47.

Goloboff, P.A. (1993). Nona. Ithaca, USA: Cladistics. Retrieved from www.cladistic.com

Hasing, R.M.E. (2004). Estudio de la variación en los contenidos de polifenoles y alcaloides en almendras de cacao por efecto de los procesos de fermentación y tostado (Tesis de grado). Escuela Superior Politécnica de Chimborazo, Riobamba, Ecuador.

Hernández-Gómez, E., Hernández-Morales, J., AvendañoArrazate, C.H., López-Guillen, G., Garrido-Ramírez, E.R., Romero-Nápoles, J., \& Nava-Díaz, C. (2015). Factores socioeconómicos y parasitológicos que limitan la producción del cacao en Chiapas, México. Revista Mexicana de Fitopatología, 33(2), 232-246.

Hii, C.L., Law, C.L., Suzannah, S., \& Cloke, M. (2009). Polyphenols in cocoa (Theobroma cacao L.). Asian Journal of Food and Agro-Industry, 2(4),702-722.

Karim, A.A., Azlan, A., Ismail, A., Hashim, P., \& Abdullah, N.A. (2014). Antioxidant properties of cocoa pods and shells. Malaysian Cocoa Journal, 8, 49-56.

Lattanzio, V., Lattanzio, V.M.T., \& Cardinali, A. (2006). Role of phenolics in the resistance mechanisms of plants against fungal pathogens and insects. Phytochemistry. Advances in Research, 37, 23-67.

McCoy, J.A., Young, J.H., Nifong, J.M., Hummer, K., DeNoma, J., Avendaño, A.C.H., ... Kantar, M.B. (2019). Species for Medicinal and Social Use with an Emphasis on Theobroma cacao L. (Cacao), Nicotiana tabacum L. (Tobacco), Actaea racemosa L. (Black Cohosh), and Humulus lupulus L. (Hops). In S. Greene, K. Williams, C. Khoury, M.B. Kantar, \& L. Marek (Eds.), North American Crop Wild Relatives (Volume 2, pp. 645-692). Suiza: Springer International Publishing.
Niemenak, N., Rohsiusb, C., Elwersb, S., Ndoumoua, D.O., \& Liebereib, R. (2006). Comparative study of different cocoa (Theobroma cacao L.) clones in terms of their phenolics and anthocyanins contents. Journal Food Composition Analysis, 19(6-7), 612-619.

Nixon, K.C. (2002). WinClada. Ithaca, USA: Cladistics. Retrieved from www.cladistic.com

Orfa, N., Ordoñez, E., Mandujano, Y., \& Arévalo, J. (2013). Polifenoles Totales, Antocianinas, Capacidad Antioxidante De Granos Secos y Análisis Sensorial Del Licor De Cacao (Theobroma cacao L.) Criollo y Siete Clones. Investigación y Amazonía, 3(1), 51-56.

Othman, A., Mail, A., Abdul, N., \& Adenan, I. (2007). Antioxidant capacity and phenolic content of cocoa beans. Food Chemistry, 100(4),1523-1530. DOI: 10.1016/j.foodchem.2005.12.021

Padilla, F.C., Rincón, A.M., \& Bou-Rached, L. (2008). Contenido de polifenoles y actividad antioxidante de varias semillas y nueces. Archivos Latinoamericanos de Nutrición, 58(3), 303-308.

Pallares-Pallares, A., Perea-Villamil, J.A., \& López-Giraldo, L.J. (2016). Impacto de las condiciones de beneficio sobre los compuestos precursores de aroma en granos de cacao (Theobroma cacao L) del clon $\mathrm{CCN}$ 51. Respuestas, 21(1), 120-133.

Perea, V.J.A., Cadena, C.T., \& Herrera, A.J. (2009). El cacao y sus productos como fuente de antioxidantes: Efecto del procesamiento. Revista Salud UIS, 41(2), 128-134.

Pourcel, L., Routaboul, J.M., Cheynier, V., Lepiniec, L., \& Debeaujon, I. (2007). Flavonoid oxidation in plants: from biochemical properties to physiological functions. Trends and Plants Science, 12(1), 29-36. DOI: $10.1016 /$ j.tplants.2006.11.006

Quiñones, G.J., Trujillo, S.R., Capdesuñer, R.Y., Quirós, M.Y., \& Hernández, T.M. (2013). Potencial de actividad antioxidante de extractos fenólicos de Theobroma cacao L. (cacao). Revista Cubana de Plantas Medicinales, 18(2), 201-215.

Ramírez, M.B., Cely, V.H., \& Ramírez, S.I. (2013). Actividad antioxidante de clones de cacao (Theobroma cacao L.) finos y aromáticos cultivados en el estado de Chiapas-México. Perspectivas en Nutrición Humana, 15, 27-40.

Re, R., Pellegrini, N., Proteggente, A., Pannala, A., Yang, M., \& Rice-Evans, C. (1999). Antioxidant activity applying an improved ABTS radical cation decolorization assay. Free Radical Biology and Medicine, 26(98), 1231-1237.

Rivera-Fernández, R.D., Mecías-Gallo, F.W., GuzmánCedeño, A.M., Peña-Galeas, M.M., Medina-Quinteros, H.N., Casanova-Ferrín, L.M., ... Nivela-Morante, P.E. (2012). Effect of time and type of fermentation in 
physical and chemical quality of cocoa (Theobroma cacao L.) national type. Ciencia y Tecnologia, 5(1), 7-12.

Rondón, B.J., \& Cumana, C.L.J. (2005). Revisión taxonómica del género Theobroma (Sterculiaceae) en Venezuela. Acta Botánica Venezuelica, 28(1), 113-133.

SAS (2001). Institute Inc., SAS/ETS 9.2. User guide. SAS, Cary, USA. Retrieved from http://morgan.dartmouth. edu/Docs/sas92/support.sas.com/documentation/cdl/ en/biig/60946/PDF/default/biig.pdf

Servicio de Información Agroalimentaria y Pesquera (2020). Avance de Siembras y Cosechas. Resumen nacional por cultivo. Retrieved from http://infosiap. siap.gob.mx:8080/agricola siap gobmx/AvanceNacionalSinPrograma.do;jsessionid=17439EA518D5B CA149E95459C0A458E5

Singleton, V.L., \& Rossi, J.A. (1965). Colorimetric of total phenolic whit phosphomolybdic-phosphotungstic acid regents. American Journal of Enology and Viticulture, 16(3), 144-158.

Sousa-Silva, C., \& Figueira, A. (2005). Phylogenetic analysis of Theobroma (Sterculiaceae) based on Kunitz-like trypsin inhibitor sequences. Plant Systematics and Evolution., 250, 93-104. DOI: 10.1007/ s00606-004-0223-2

Suárez-Venero, G.M., Avendaño-Arrazate, C.H., RuízCruz, P.A., \& Estrada-de los Santos, P. (2019). Tree diversity and stored carbon in cocoa (Theobroma cacao L.) agroforestry systems in Soconusco, Chiapas, Mexico. Revista Chapingo Serie Ciencias Forestales, 25(3), 1-18. DOI: 10.5154/r. rchscfa.2018.12.093
Valadez-Carmona, L., Plazola-Jacinto, C.P., HernándezOrtega, M., Hernández-Navarro, M.D., Villarreal, F., Necoechea-Mondragón, H., ... Ceballos-Reyese, G. (2017). Effects of microwaves, hot air and freezedrying on the phenolic compounds, antioxidant capacity, enzyme activity and microstructure of cacao pod husks (Theobroma cacao L.). Innovative Food Science \& Emerging Technologies, 41, 378-386. DOI: $10.1016 /$ j.ifset.2017.04.012

Vázquez-Ovando, A., Molina-Freaner, F., Nuñez-Farfán, J., Betancur-Ancona, D., \& Salvador-Figueroa, M. (2015). Classification of cacao beans (Theobroma cacao L.) of southern Mexico based on chemometric analysis with multivariate approach. European Food Research and Technology, 240, 1117-1128.

Wang, P., Mu, X., Du, J., Gao, Y., Bai, D., Jia, L., ... Xue, X. (2018). Flavonoid content and radical scavenging activity in fruits of Chinese dwarf cherry (Cerasus humilis) genotypes. Journal of Forestry Research, 29, 55-63. DOI: $10.1007 / \mathrm{s} 11676-017-0418-3$

Waśkiewicz, A., Muzolf-Panek, M., \& Goliński, P. (2013). Phenolic Content Changes in Plants Under Salt Stress. In P. Ahmad, M. Azooz, \& M. Prasad (Eds.), Ecophysiology and Responses of Plants under Salt Stress (pp. 283-314). New York, USA: Springer.

Waterman, P.G., \& Mole, S. (1994) Analysis of Phenolic Plant Metabolites. Oxford, UK: Blackwell Scientific Publications.

Zapata-Bustamante, S., Tamayo-Tenorio, A., \& AlbertoRojano, B. (2013). Efecto de la fermentación sobre la actividad antioxidante de diferentes clones de cacao colombiano. Revista Cubana de Plantas Medicinales, 18(3), 391-404. 